\title{
COZUMEL Y LA TRANSFORMACIÓN DE SU PAISAJE POR EL TURISMO DE CRUCEROS
}

\section{COZUMEL' ISLAND TRANSFORMATION BY TOURISM CRUISE SHIP}

\author{
Alejandro Palafox Muñoz* \\ Arturo Aguilar Aguilar \\ Julia Sderis Anaya Ortiz ${ }^{* * *}$
}

RESUMEN

El artículo hace referencia al crecimiento económico del sistema insular provocado por la transformación del paisaje para beneficio de la actividad turística, mediante la comercialización de productos y servicios a escala global, en el marco del nuevo colonialismo. El artículo es el resultado de una investigación vinculada al análisis de las alteraciones en la costa occidental de la isla de Cozumel, provocadas por el impulso al turismo de cruceros. Lo anterior permite revelar el proceso de homogenización del espacio turístico insular con impactos en el espacio a raíz de los cambios en el modo de producción y la dependencia económica de la zona.

PALABRAS CLAVE: MÉXICO * TURISMO * PAISAJE * TRANSFORMACIÓN * CAPITALISMO

\section{ABSTRACT}

The article is about the economic growth of the insular system caused by the transformation of the land scape for the benefit of tourism through the marketing products and services offered globally, under the context of new colonialism. The article is the result of an investigation related to the analysis of alterations in the west coast of the island of Cozumel, caused by the impulse of cruise tourism. This can reveal the process of homogenization of insular space tourism impacts in space as a result of changes in the mode of production and the economic dependence of the area.

KEYWORDS: MEXICO * TOURISM * LANDSCAPE * TRANSFORMATION * CAPITALISM

Universidad de Quintana Roo, México.

alejandro.palafox@gmail.com

** Universidad de Quintana Roo, México. aguilarr2d2@gmail.com

Universidad de Quintana Roo, México. juliasderis@gmail.com 


\section{INTRODUCCIÓN}

Los países y regiones que cuentan con recursos naturales y culturales son de interés para el desarrollo de la actividad turística sobre todo de índole internacional, aun cuando las economías incipientes no cuentan con el capital suficiente para promoverlo, en un panorama que aprovechan los Organismos Internacionales (oI) para impulsar empréstitos para la creación de infraestructura de acceso y servicios que necesitan las comunidades rurales, con el fin de facilitar la llegada de las inversiones privadas y configurar el paisaje para el desarrollo turístico. Estos organismos como el Fondo Monetario Internacional (FMI), el Banco Mundial (вм), el Banco Interamericano para el Desarrollo (BID) y la Organización para la Cooperación y el Desarrollo Económico (ocDE) sugieren la aplicación de políticas que beneficien la atracción de la inversión, entre las que destacan: a) la reducción de costos, b) disminución del gasto público y c) la flexibilización del empleo (Palafox, Zizumbo y Arriaga, 2010).

La riqueza natural y cultural, así como, el financiamiento de la actividad turística son factores determinantes en el crecimiento económico de un territorio, por ejemplo, en la región del Caribe, el turismo es la principal actividad económica dominante de alta demanda, ya que dicha área contribuye en promedio con el 14,5\% del Producto Interno Bruto (PIB), ello posiciona a esta zona geográfica como la de mayor dependencia económica hacia un sector de la economía (Bolaky, 2011). El éxito de la región se debe a la calidad del paisaje, el cual es considerado como elemento natural de alta valoración que motiva el desplazamiento, en ese sentido, el litoral costero se constituye como elemento protagónico del quehacer turístico $y$ de otras actividades económicas (Benseny, 2006), con lo cual, el Caribe recibió la llegada de 21,2 millones de turistas extranjeros $(2,8 \%$ más que el año 2012) (World Tourism Organization-wто, 2014).

En el caribe mexicano se encuentra la isla de Cozumel, la cual es considerada un destino turístico de tipo tradicional, ya que no surgió como resultado de los Centros Integralmente Planeados, sino que su origen espontáneo fue previo a la aparición de Cancún, Huatulco, Loreto, Los Cabos e Ixtapa, en el marco de la planeación del turismo a cargo del Fondo Nacional de Fomento al Turismo (FonatuR). El natural crecimiento del sistema insular derivó en el incremento poblacional de las últimas décadas, teniendo como resultado que el $52,5 \%$ de la población no es nativa (Instituto Nacional de Estadística y GeografíaINEGI, 2010). Lo anterior contribuye en la trasformación del paisaje, tanto para beneficio de la actividad turística como de la población.

En este sentido, el turismo es la primera actividad económica en la isla, específicamente en atención a dos segmentos de mercado distintos: el crucero y el buceo. De esta manera, en el año 2013 se recibieron un total de 2753608 pasajeros de crucero y 447747 turistas; la derrama económica del sector turístico de pernocta aportó $\$ 240,88$ millones de dólares (MDD) y el relacionado con los cruceros $\$ 225,80$ mDD. Asimismo, en Cozumel, la práctica turística se ha diversificado y el cambio es dinámico, sobre todo en la Zona de Apropiación Turística Centro [zat Centro] (Palafox, Madrigal y Zizumbo, 2011), dejando en claro que el turista de pernocta contribuye con el 51,7\% de las divisas con solo el 13,9\% de la demanda, por lo que su aporte en la derrama económica es más significativa.

\section{LA ISLA DE COZUMEL, SU PAISAJE Y ACTIVIDAD TURÍSTICA}

Cozumel se localiza en la costa oriental de la Península de Yucatán, su territorio tiene 45 kilómetros de largo y 17 kilómetros de ancho (Gómez, 1998). En la isla predominan las estructuras bajas compuestas de suelo calizo y pedregoso (Xacur, 1998). Por las características físicas del terreno compuesto por caliza, se impide los escurrimientos superficiales, por ello, el suministro de agua se realiza a través del acuífero ubicado en la porción central del territorio insular (Martínez, 2007).

Al inicio de la década de 1920, la economía de la isla estaba fundamentada en el sector primario, principalmente por la exportación de chicle y copra. En el poblado de El Cedral existían hortalizas localizadas en las casas de los 
residentes del pueblo y la propiedad de la tierra era ejidal. La zona urbano-rural se ubica en San Miguel de Cozumel, su paisaje estaba cons- tituido por extensiones de playa con cocales $y$ uvas de mar (fotografía 1), así como calles con brechas de arena y saskab (Palafox, 2011).

FOTOGRAFÍA 1

PAISAJE DE LA ISLA DE COZUMEL 1920

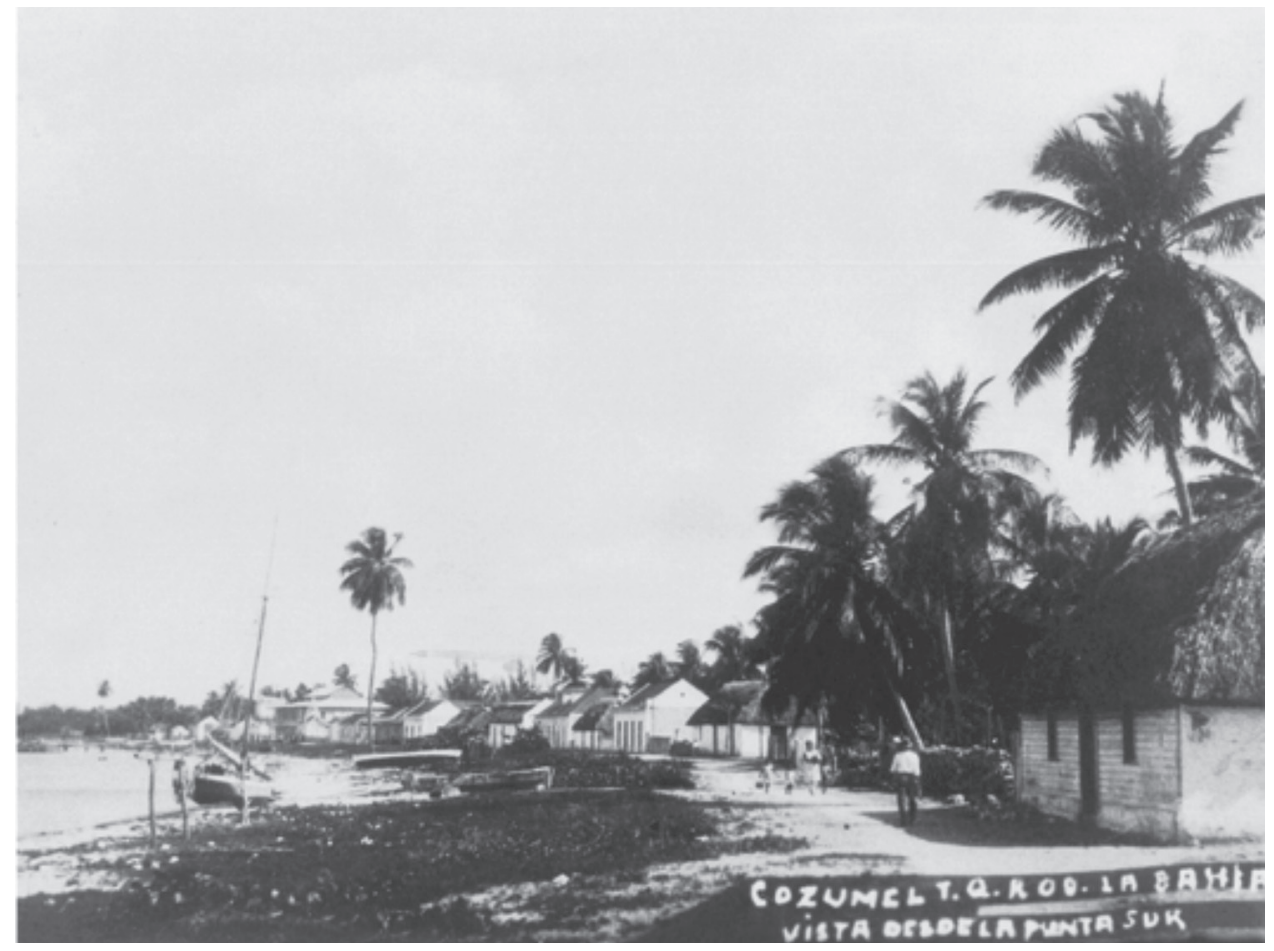

Fuente: Museo de la Isla de Cozumel.

Hasta la segunda década del siglo xx, los viajes se hacían en canoas y balandros de vela hasta los puertos del norte de Yucatán, en ese entonces la travesía duraba hasta una semana. Los únicos puertos en el territorio eran los de Puerto Juárez, Puerto Morelos, Playa del Carmen, X'calak y Payo Obispo, todos ellos aislados pues no se contaba con caminos de acceso; sin embargo, la ampliación de la red carretera llegó a Playa del Carmen y en los 70, se comenzaba el camino a Tulum y Felipe Carrillo Puerto, por lo que la nueva conexión a Cozumel es desde Playa del Carmen, ya que anteriormente el traslado marítimo a la isla era desde Puerto Morelos. Con la nueva ruta aumentó el número de pasajeros como respuesta a la construcción del muelle, el cual fue edificado mediante la inversión del empresario Trinidad Molina Cásares de origen yucateco (Vivas, 2008). La conectividad aérea era por medio de hidroaviones de Pan Am cuya ruta era de Key West a Colón, Panamá con escala en Cozumel.

Al involucrarse México en la Segunda Guerra Mundial como aliado contra los países totalitarios, provocó que en 1943 se construyera un aeropuerto mediante un acuerdo con los Estados Unidos de Norteamérica, las pistas que ahora son utilizadas por la Fuerza Aérea Mexicana y la aviación comercial. De esta manera, después de la guerra, Transportes Aéreos Mexicanos SA (TAMsA) volaba con dirección 
a Mérida y Chetumal (Ibíd.), posteriormente, Mexicana de Aviación consolida los vuelos a Miami y Ciudad de México; actualmente, se cuenta con vuelos directos hacia $y$ desde el Distrito Federal a través de aerolíneas Interjet.

El buceo y el turismo de cruceros son los dos segmentos de mercado que recibe Cozumel, en ese sentido, el arribo de turistas y visitantes se plasma en el gráfico 1 . Por el número de pasajeros, se ha determinado gran parte de la oferta turística de la zat Centro de la ínsula, como resultado de la consolidación de esa porción de visitantes. Sin embargo, su derrama económica no es tan sólida en comparación con la generada por la corriente turística de pernocta, este segmento aporta en promedio más del $50 \%$ de la derrama económica de la localidad en la última década $(56,5 \%)$.

GRÁFICO 1

AFLUENCIA TURÍSTICA A COZUMEL $2000-2013$

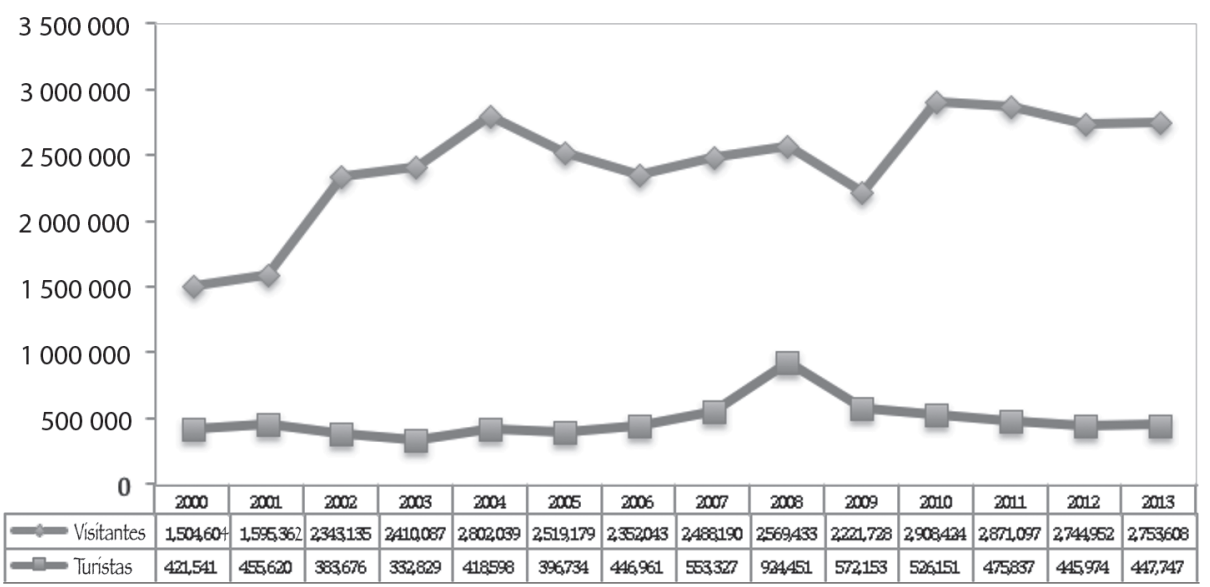

Fuente: Elaboración propia a partir de los datos de la Secretaría de Turismo del Estado de Quintana Roo, 2000-2013.

El número que representa el arribo de pasajeros de cruceros ha tenido un comportamiento similar durante los últimos doce años, en promedio llegan 2,4 millones de personas vía marítima y 0,48 millones de turistas que pernoctan en la localidad. Asimismo, la derrama económica global es de $\$ 2628,68$ мDD para el segmento de cruceros y de $\$ 3418,68$ mDD para el turismo de pernocta (gráfico 2). Sin embargo, la promoción del turismo de la isla de Cozumel, está fuertemente dirigida hacia la atracción de cruceros, ya que a partir de la década de los 90, la construcción de infraestructura portuaria ha sido notable, por lo que ahora la isla cuenta con tres muelles para arribo de turistas: Puerta Maya (Carnival Cruises), Muelle Internacional (Stevedoring Services of America) y Punta Langosta (Grupo Aviomar); un muelle para transporte de mercancías (Administración Portuaria Integral de Quintana Roo) y otro para la ruta Cozumel -Playa del Carmen-Cozumel (Muelle Fiscal). 


\section{GRÁFICO 2 \\ DERRAMA ECONÓMICA \\ 2000-2013}

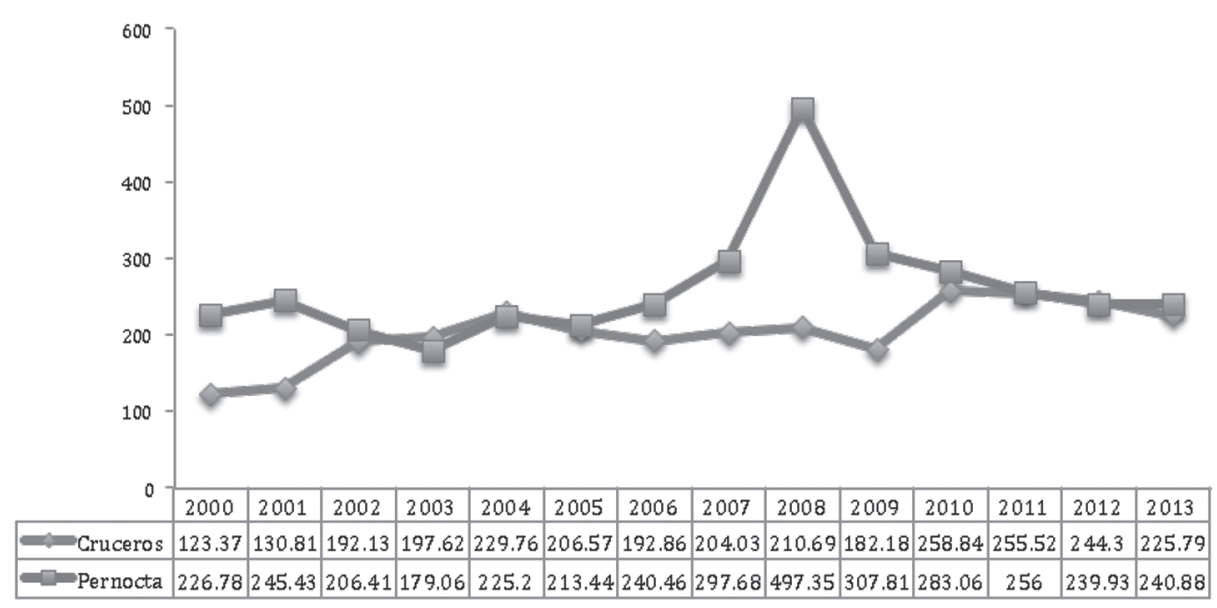

Fuente: Elaboración propia a partir de los datos de la Secretaría de Turismo del Estado de Quintana Roo, 2000-2013.

Los flujos de turismo internacional que se presentaron anteriormente confirman que la actividad turística fue más accesible a la clase media, lo que llevó a la masificación de la actividad. Desde entonces, el crecimiento acelerado de esta industria fue acompañado con la progresiva complejidad y sofisticación de sus prácticas comerciales, la deslocalización de los destinos hacia los países en desarrollo y la creciente diversificación de la oferta (Fernández, 2012). Una clave de éxito de la industria turística de cruceros es que se visualizó como un resort flotante $y$ no como transporte, aunque finalmente cumple ambas funciones. Además, se incluyen en la oferta el entretenimiento de casino, con el cual tomó un mayor impulso este segmento de mercado turístico (Organización Mundial de Turismo-омт, 2008).

En otras palabras, la oferta se ha adaptado a las demandas del turismo en lugar de la excesiva rigidez que se imponía en la época de los viajes trasatlánticos. Al crecer en tamaño y número de embarcaciones año con año, se pueden ofrecer más espacios a menor costo $y$ al mismo tiempo, más sitios que visitar, por lo cual se necesita invertir en infraestructura portuaria en los destinos con el impacto al paisaje $y$ a la comunidad receptora. En este sentido, el turismo de cruceros se consolida como "un mercado con un gran dinamismo, una actividad con una creciente concentración económica, un fuerte desarrollo tecnológico, y una flexibilidad operativa y elusión normativa" (Fernández, 2012: 5). Por su ubicación en el Caribe, Cozumel se consolida como punto estratégico para el desarrollo de la industria naviera recreativa.

\section{LA TRANSFORMACIÓN DEL PAISAJE PARA EL DESARROLLO TURÍSTICO}

Dos Santos menciona la importancia de la relación entre paisaje y turismo, la cual está "delineada a partir del reconocimiento del paisaje como un ente indisociable del turismo... y es un recurso de gran valor para el desarrollo y la consolidación de la oferta" (2011: 522-523). El paisaje, es un elemento que utiliza la industria turística para motivar el desplazamiento de personas, aunque la relación de ese lugar turístico con sus residentes, sea distinta a la que el turista espera ante el cambio de escenario, el visitante 
"encontrará en el paisaje el elemento que mejor le indicará esa tan deseada ruptura con la percepción visual de lo nuevo y diferente en el transcurso de su experiencia de viaje" (Nogué, 1989 citado en Dos Santos, 2011: 523). En este sentido, el litoral costero cobra relevancia por sus características naturales, las playas cuentan con gran valoración por parte del visitante (Benseny, 2006), asumiendo gran protagonismo para el desarrollo de la actividad turística, por lo que los países con características similares se vuelven objeto de inversión de los estados económicamente desarrollados.

De acuerdo con Moraga (2008), en el paisaje existen formas y estructuras de dominio y subyugación asociadas a conceptos funcionalistas, sobre todo reflejan las formas de valoración económica del espacio. Así, se transforma la costa en un complejo turístico propicio para la llegada de un turista estandarizado en busca de lo que tiene en su país, de ahí el proceso de apropiación, funcionalización $y$ homogenización del espacio para el desarrollo del turismo (Palafox, Madrigal y Zizumbo, 2011), principalmente en localidades pobres, sin servicios e infraestructura pero con gran riqueza natural $y$ cultural.

Por lo tanto, los destinos turísticos no se despojan de la imagen polarizada de las ciudades contemporáneas, sino la intensifican desde la dimensión urbana, por lo cual los enclaves costeros para el turismo construyen paisajes segregados y fragmentados de las ciudades, inhibiendo la planeación y la integración urbana. Por ello, la construcción de establecimientos de hospedaje, marinas, fraccionamientos residenciales y campos de golf generan un deterioro ambiental importante sobre las zonas de humedales, manglares $y$ dunas costeras, dando lugar a paisajes artificiales, mismos que generan un acelerado crecimiento urbano y demográfico (Enríquez, 2008).

Como se mencionó con anterioridad, para la industria turístico-recreativa, los recursos naturales son de alto valor paisajístico y económico, por ello, el capital se apropia de ellos para transformarlos, de ahí la importancia de analizar las relaciones en el espacio antropogénico, debido a las relaciones dialécticas que se suscitan en el entorno. Existe una contradicción entre la capacidad de tratar el territorio a gran escala y la propiedad privada del área, por lo que esa producción del espacio es compleja, por lo tanto es imprescindible una planificación con el fin de resolver esa composición (Lefebvre, 1974), misma que tiene su origen en la propiedad privada $y$ la libertad financiera para el desarrollo del modo de producción capitalista, así:

...el fenómeno de la internacionalización económica se caracteriza por el crecimiento en los flujos de mercancías, capital y tecnología, lo cual ocurre en contextos y relaciones imperialistas durante las últimas décadas y se ha enfatizado con la firma de los acuerdos para la explotación de recursos humanos y materiales de la periferia capitalista (Palafox, Zizumbo, Arriaga y Monterroso, 2010: 463).

De acuerdo con Harvey (1977), son cuatro los factores que influyen para la transformación del paisaje: a) el sistema financiero globalizante, b) la revolución informática y tecnológica, c) la reducción de los costos de transporte y d) mano de obra; por lo tanto, "el capital por naturaleza, crea unos ambientes físicos a su imagen y semejanza, solamente para destruirlos más adelante, cuando busque expansiones geográficas y desubicaciones temporales, en un intento de solucionar las crisis de sobreacumulación que lo afectan cíclicamente" (Harvey, 2004: 4). En este mismo sentido, la apertura de nuevos mercados no abre la competencia, sino ofrece nuevas oportunidades de expansión a los poderes monopolistas, así como un sin límite de consecuencias sociales, ecológicas, económicas y políticas, teniendo como resultado una desigualdad generalizada (Harvey, 2004).

El factor económico ha sido dominante en el desarrollo de los países capitalistas emergentes. De esta manera a través de la comercialización global de productos $y$ servicios se han abierto fronteras, se ha dado el crecimiento del turismo y se ha intervenido el paisaje, así como, las sociedades han sufrido transformaciones espaciales, a lo que Harvey 
(2000) denomina nuevo colonialismo por cuatro factores principales:

1) A través de la desregulación del capital al interior y entre países, el desarrollo del sistema financiero ha adquirido poder sobre otros sectores productivos, es decir que ahora el sistema financiero no se preocupa por producir objetos sino que produce únicamente dinero; este sistema tiene un rol determinante entre las diferentes regiones del mundo.

2) La revolución informática ha acelerado las comunicaciones entre los países.

3) La reducción de los costos de transporte ha creado una gran movilidad entre las regiones, ello ha influenciado al turismo de masas y las migraciones en busca de empleo en el sector turístico.

4) Lo que se denomina la clase trabajadora global, se ha convertido en una expansión del proceso de proletarización a tal grado que ha duplicado su número, debido al proceso natural del crecimiento de la población y por la absorción de cada vez más gente como mano de obra.
Estos factores se conjugan para fortalecer la política económica global, asimismo han influido en las transformaciones del espacio e inciden sobre las directrices del desarrollo del turismo en el mundo, para la acumulación del capital (Palafox y Zizumbo, 2009; Palafox, Zizumbo y Arriaga, 2010; Palafox, Madrigal y Zizumbo, 2011 y Palafox, 2013) a través de los grandes proyectos turísticos en las regiones de los países en vías de desarrollo, por medio de la globalización como estrategia económica para la difusión del nuevo eje de acumulación.

\section{EL TURISMO DE CRUCEROS Y SU IMPACTO EN COZUMEL}

La industria de cruceros ha crecido a un ritmo sostenido por los últimos años, sobre todo en la zona del Caribe, en donde las rutas se implementan prácticamente todo el año, ello ha permitido el crecimiento de la industria en la zona (Tabla 1), asimismo, la temporalidad del viaje ha ido en aumento, en donde las travesías de 6 a 8 días ocupan el 53,3\% de los viajes (Cruise Line International Association Inc-cLIA, 2011).

TABLA 1

DESTINO DE CRUCERISTAS AMERICANOS Y CANADIENSES (MILES DE PASAJEROS)

2001-2005

\begin{tabular}{lcc|cc|cc|cc}
\hline \multirow{2}{*}{ Zona } & \multicolumn{2}{c|}{2001} & \multicolumn{2}{c|}{2002} & \multicolumn{2}{c|}{2003} & \multicolumn{2}{c}{2005} \\
\cline { 2 - 9 } & CRUCERISTAS & $\%$ & CRUCERISTAS & $\%$ & CRUCERISTAS & $\%$ & CRUCERISTAS & $\%$ \\
\hline $\begin{array}{l}\text { El Caribe / } \\
\text { Bahamas }\end{array}$ & 4663 & 70,4 & 5433 & 71,1 & 5966 & 72,0 & 6713 & 70,4 \\
\hline Transcanal & 117 & 1,8 & 96 & 1,3 & 91 & 1,1 & 114 & 1,2 \\
Alaska & 678 & 10,2 & 722 & 9,4 & 766 & 9,2 & 858 & 9,0 \\
Pacífico Mexicano & 611 & 9,2 & 627 & 8,2 & 731 & 8,8 & 1076 & 11,3 \\
Hawaii & 71 & 1,1 & 231 & 3,0 & 222 & 2,7 & 192 & 2,0 \\
Bermuda & 167 & 2,5 & 190 & 2,5 & 207 & 2,5 & 194 & 2,0 \\
Canadá/ & 154 & 2,3 & 158 & 2,1 & 169 & 2,0 & 214 & 2,2 \\
New England & & & & & & & & \\
\hline TOTAL
\end{tabular}

Fuente: Organización Mundial del Turismo-omT, 2008. 
El mercado americano es el de mayor demanda en la región del Caribe; sin embargo, desde el 2008, la mirada de China se está dirigiendo a esta región del Caribe.

En la isla de Cozumel, el turismo de cruceros ha sido promovido desde hace tres décadas y el crecimiento ha sido paulatino, también es perceptible la baja que presenta desde hace nueve años, atribuida a varios factores, principalmente, a la recesión económica estadounidense (2009), la dependencia económica y de visitantes de un solo país de origen, además que poco más del 80\% de las navieras son de origen norteamericano (омт, 2008), así como, los ciclones tropicales de Emily y Wilma en el año 2005 (Palafox y Frausto, 2008) y la epidemia de aH1N1 (2009).

GRÁFICO 3

\section{EVOLUCIÓN DEL TURISMO DE CRUCEROS}

1981- 2013

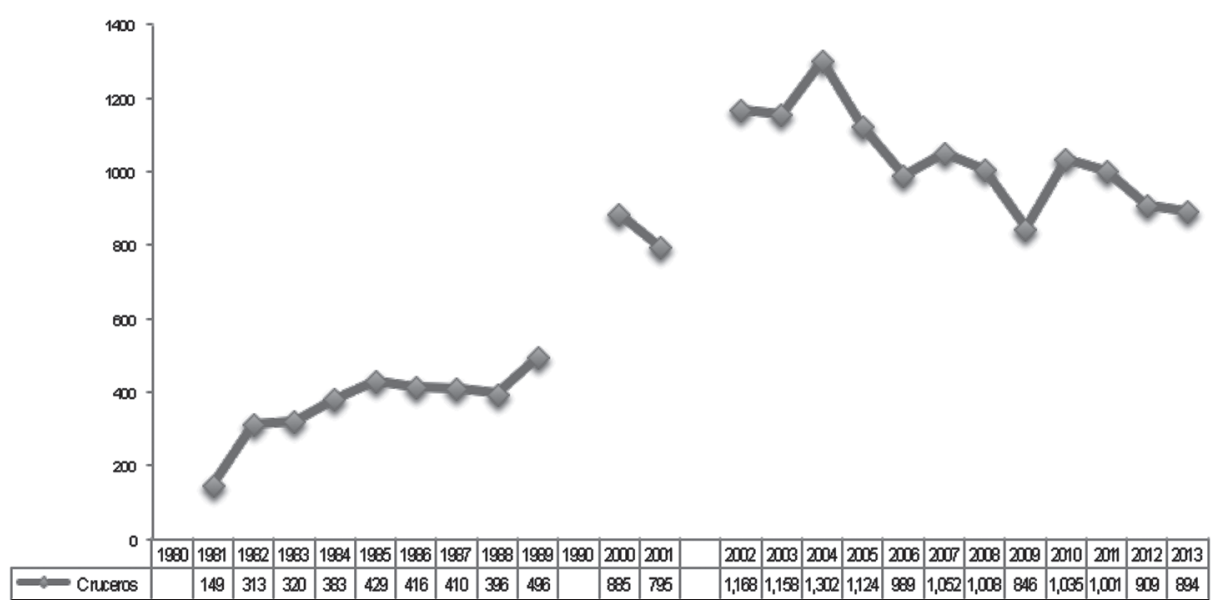

Fuente: Martínez, 2007 y Secretaría de Turismo del Estado de Quintana Roo (www.sedetur.qroo.gob.mx).

Con el paso del tiempo, la transformación del paisaje de Cozumel es notoria, principalmente en la zAT Centro, como consecuencia de la promoción del turismo de cruceros, el cual desde la década de los 80 presenta un crecimiento sostenido, sin embargo, a partir del año 2005, empieza un constante decremento de visitantes. No obstante, la caída en la recepción de barcos marca un claro descenso del segmento de cruceros en el país, lo que repercute en el crecimiento económico de la localidad resultado de la alta dependencia que existe en el turismo en México.

La dinámica en el cambio del paisaje para la promoción del turismo en Cozumel obedece al nuevo segmento de cruceros que tiene un crecimiento acelerado, para el desarrollo de este tipo de turismo, se ha invertido principalmente en la creación de infraestructura portuaria y la la zAT Centro ha modificado su escenario para el establecimiento de joyerías y tiendas de artesanías. Estas inversiones han atraído a otras de servicios con las que se ha transformado el litoral costero, como ejemplo de ello existe en cada muelle de cruceros una plaza comercial que da servicio directamente al turista de cruceros, en suma a las plazas, joyerías, restaurantes, rentadoras y locales comerciales que se han desarrollado en la avenida costera (fotografía 2). 


\section{FOTOGRAFÍA 2 \\ PUNTA LANGOSTA \\ 1980 Y 2014}
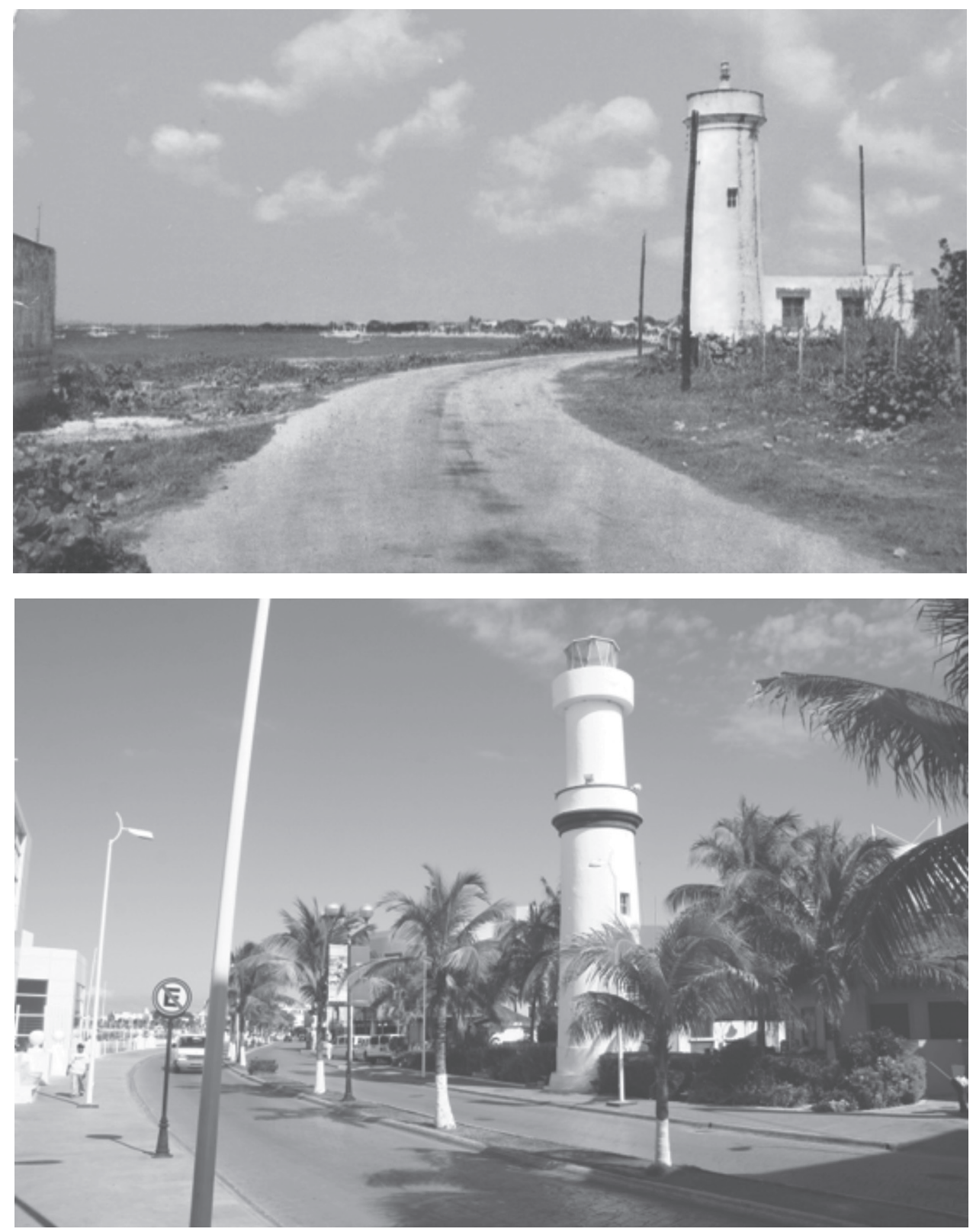

Fuente: Aguilar, 2014.

Todo ello ha llevado a un cambio en la fisonomía urbana de Cozumel, pasando de un pueblo de pescadores con una imagen homogénea en el tipo de construcción y colores de fachada, a una pequeña ciudad con una imagen abigarrada $y$ heterogénea por la importación de formas y materiales, con lo cual se ha perdido parte de la identidad de la isla. 
Las transformaciones comenzaron desde la década de los 50, principalmente sobre la zona costera donde se establece el centro urbano de la isla de Cozumel. La creación de infraestructura para el desarrollo del turismo de cruceros y la consolidación del segmento de viajeros vía marítima como una estrategia para la permanencia del modo de producción, ha captado el interés de fonatur (2008) para la creación de una marina que atienda 36 mil nuevos usuarios a través de 133 embarcaciones, estimando una captación de 45 millones de dólares anuales y acceder al segmento de escaleras náuticas (Palafox y Zizumbo, 2009).

El comercio en la isla ha modificado su esencia, en la década de los 80 y 90, el comercio sobre el malecón costero eran principalmente restaurantes, hoteles, tiendas de artesanías, entre otros; sin embargo, al comenzar la promoción intensiva del turismo de cruceros, estos espacios fueron cambiando a la venta de platería y joyería (diamantes y otros tipo de piedras preciosas y metales). En 1988, aparece la primera joyería en la Avenida Rafael E. Melgar, ello condujo a que la vida turístico-comercial de la isla esté supeditada a las condiciones de la demanda específica del visitante de cruceros. Al final de la década de los 90, tras concluir la construcción de la infraestructura portuaria dedicada al servicio de los cruceros, inicia el descenso de la derrama económica por los siguientes factores: el all inclusive de los cruceros y la concentración de la demanda en las plazas comerciales que están construidos al interior o frente a los muelles (Aguilar, 2014).

Uno de los pilares estratégicos de la globalización de la economía es la estandarización de los servicios y la imagen de los productos, para ello es necesario un proceso de apropiación del territorio con la finalidad de funcionalizarlo a través de la infraestructura de acceso $y$ servicios, con el fin de homogenizarlo para la satisfacción de las necesidades de la oferta turística. En este sentido, la isla en la década de los 80 se instaló el drenaje sanitario, entubado para la distribución de agua potable y la pavimentación de calles. Posteriormente, en el año 2005, después del impacto del huracán Wilma, se llevó a cabo la estandarización de la imagen y fisonomía urbana del centro del destino turístico, teniendo como resultado la misma oferta turística del resto de los destinos turísticos de la región del Caribe.

Finalmente, las categorías de análisis de Harvey (1977) que contribuyen a entender la transformación del destino a través del turismo de cruceros son: a) el financiamiento de la infraestructura portuaria para la recepción de un mayor número de embarcaciones; b) las comunicaciones han aumentado por miedo del uso de nuevas tecnologías, por lo que el visitante puede acceder a la compra y renta de servicios turísticos desde su hogar; c) con la modernización del transporte se desplazan cada vez más turistas a los destinos turísticos, el contar con más de una alternativa de transportación se promueve el abaratamiento del mismo y d) la mano de obra ha requerido de especialización para la atención del visitante, provocando altas concentraciones en las zonas urbanas y dejando el campo abandonado. De esta manera el turismo se promueve como detonante del crecimiento económico y desarrollo social, no obstante, los beneficios sociales son mínimos.

\section{CONCLUSIONES}

Desde la década de los 70, en México, el turismo se propuso como una actividad económica que promueve el crecimiento y el desarrollo regional, sin embargo, tales bondades no han sido del todo visibles en las localidades donde se ha implementado como instrumento que no solo desarrolla sino apoya el progreso de la industria.

El estado de Quintana Roo ha recibido los beneficios del financiamiento de los Organismos Internacionales a partir de la creación del Centro Integralmente Planeado de Cancún para el establecimiento de infraestructura de acceso y servicios, asimismo para el Megaproyecto Turístico de Riviera Maya, Costa Maya y para la Marina Cozumel.

Para ello, fue necesaria la apropiación del territorio, una vez logrado lo anterior, el proceso de funcionalización y con los préstamos internacionales fue tomando forma para comenzar el proceso de homogenización de los destinos, a fin de satisfacer la demanda de los 
visitantes, principalmente de Estados Unidos de Norteamérica, logrando una dependencia económica de la región, ya que los sectores primario y secundario carecen de apoyo por parte del Estado.

En este sentido, la teoría de la transformación del paisaje de David Harvey (1977) proporciona las categorías de análisis para el estudio del cambio en el entorno para el crecimiento y expansión de una actividad económica que por sus características es considerada un eje de acumulación, ya que busca la reproducción del modo de producción capitalista.

Los cruceros turísticos son el segmento de mercado que promueve el Estado; sin embargo, en este proceso de transformación del espacio, la zona costera de la isla ha ido cambiando en primera instancia, el uso del suelo y posteriormente, su valor paisajístico, el cual con la creación de infraestructura para el desarrollo turístico adquiere un valor de tipo económico, de tal forma que la infraestructura es de carácter privado.

Las cifras indican que la demanda turística relacionada con los cruceros genera una importante derrama económica, a un alto costo por la transformación que necesitó el territorio para convertirse en un destino turístico de esta índole. Tal apoyo a la industria naviera de tipo recreativo ha minimizado el quehacer del otro segmento turístico que busca la riqueza natural de la localidad (buceo) y que con una demanda limitada contribuye sustancialmente a la economía local. Asimismo, la actividad económica insular se ha adecuado a los tiempos relacionados con los cruceros, de tal forma que no se aperturan los comercios de la zAT Centro sino hay barco en puerto.

La conciencia turística relacionada con la conservación de los arrecifes ha disminuido, por el interés en el arribo de millones de turistas vía crucero, ello ha provocado la masificación de la actividad, saturando el destino y los espacios recreativos, teniendo en consecuencia la transformación del paisaje para la satisfacción de una demanda específica y sin cultura, cuyo fin último es de carácter hedónico (Anaya y Palafox, 2010).
Los comercios instalados en las Plazas Comerciales anexas a los muelles, son la principal evidencia del proceso de apropiación, funcionalización y homogenización del espacio para el turismo, solo a través del trabajo de campo se pueden constatar las mismas tiendas de anteojos, joyas, perfumes, bares, entre otros; es decir, el visitante compra las marcas que reconoce en su país de origen, lo anterior deriva en el deterioro del comercio de firmas locales, como resultado del proceso de homogenización cultural, este proceso contribuye a que el modo de producción capitalista sea visto como un modo de vida.

En este sentido, la política turística está vinculada a la obtención de divisas, aun cuando en el discurso tenga una visión sustentable. Los impactos sociales y ambientales del turismo de cruceros, distan de contribuir a alcanzar un desarrollo, aunque su impacto académico sea visible, queda en duda, cuál es el destino final de los recursos económicos por concepto de turismo de cruceros, toda vez que las navieras y muelles son en su mayoría de capitales extranjeros.

\section{BIBLIOGRAFÍA}

\section{LIBROS}

Gómez, L. La isla de Cozumel. México: Editorial Kukulkán, 1998.

Harvey, David. Urbanismo y desigualdad social. $1^{\text {a }}$ Edición. España: Siglo xxi, 1977.

Harvey, David. "Mundos urbanos posibles". Lo urbano en 20 autores contemporáneos. Ángel Martín Ramos (coord.). España. Universitat Polytècnica de Catalunya, 2000: 177-198.

Palafox Muñoz, Alejandro y Frausto Martínez, Oscar. Turismo: desastres naturales, sociedad y medio ambiente. México: Plaza y Valdés-Universidad de Quintana Roo, 2008.

Palafox Muñoz, Alejandro. "Pasaje y turismo en Cozumel". Ambientes del turismo: actores y escenarios. Alejandro Palafox Muñoz y Alejandro Alvarado Herrera (coords.). Chetumal, México. Universidad de Quintana Roo, 2011: 51-68.

Vivas Valdés, Velio. Travesía por la historia de Cozumel. México: H. Ayuntamiento de Cozumel, 2008. 
Xacur Maiza, Juan Ángel. Enciclopedia de Quintana Roo. Chetumal, México: Gobierno del Estado de Quintana Roo, 1998.

\section{PUBLICACIONES PERIÓDICAS}

Anaya Ortiz, Julia Sderis y Palafox Muñoz, Alejandro. "El perfil del turista internacional de Cozumel a partir de la construcción de su capital simbólico". Teoría y Praxis 8. México. Universidad de Quintana Roo, 2010: 171-185.

Benseny, Graciela. "El espacio turístico litoral”. Aportes y Transferencias 2 (10). Argentina. Universidad Nacional de Mar del Plata, 2006: 102-122.

Bolaky, Bineswaree. "La competitividad del turismo en el Caribe". Revista CEPAL 104. Chile. Comisión Económica para América Latina y el Caribe, 2011: 55-79.

Dos Santos Pires, Paulo. "Marco teóricometodológico de los estudios de paisaje". Estudios y Perspectivas en Turismo 20 (3). Argentina. Centro de Investigaciones y Estudios Turísticos, 2011: 522-541.

Fernández Miranda, Rodrigo. "Lo que hunden mientras flotan”. Alba Sud 13. Alba Sud. Investigación y Comunicación para el Desarrollo, 2012: 2-42.

Harvey, David. "El nuevo imperialismo: sobre reajustes espacio temporales $y$ acumulación mediante desposesión". Viento Sur 447. España. Viento Sur, 2004: 1-26.

Lefebvre, Henri. "La producción del espacio". Papers Revista de Sociologia 3. España. Universitat Autònoma de Barcelona, 1974: 219-229.

Moraga Peralta, Julio César. "Globalización del paisaje en Costa Rica y la importancia de los sistemas de información geográfica en su interpretación". Tecnología en marcha 21 (1). Costa Rica. Instituto Tecnológico de Costa Rica, 2008: 215-227.

Nogué i Font, Joan. "Paisaje y turismo". Estudios Turísticos 103. España. Instituto de Estudios Turísticos del Gobierno de España, 1989: 35-45.
World Tourism Organization (unwTo). "Strong peak season anticipated for international tourism". World Tourism Barometer 3 (12). eeuu. World Tourism Organization, 2014: 1-6.

Palafox Muñoz, Alejandro y Zizumbo Villarreal, Lilia. "Distribución territorial y turismo en Cozumel, Estado de Quintana Roo, México". Gestión Turística 11. Chile. Universidad Austral de Chile, 2009: 69-88.

Palafox Muñoz, Alejandro; Madrigal Uribe, Delfino y Zizumbo Villarreal, Lilia. "Apropiación, funcionalización y homogenización del espacio para el desarrollo turístico de Quintana Roo". Caderno Virtual de Turismo 2 (11). Brasil. Universidad Federal do Rio de Janeiro, 2011: 282-293.

Palafox Muñoz, Alejandro; Zizumbo Villarreal, Lilia y Arriaga Álvarez, Emilio. "El turismo como eje de acumulación: el caso del sector hotelero en México". Multiciencias 10 (2). Venezuela. Universidad del Zulia, 2010: 193-201.

Palafox Muñoz, Alejandro; Zizumbo Villarreal, Lilia; Arriaga Álvarez, Emilio y Monterroso Salvatierra, Neptalí. "Introducción al estudio del turismo a través del materialismo cultural". Polis 25. Chile. Universidad de los Lagos, 2010: 461-486.

Palafox Muñoz, Alejandro. "El turismo como eje de acumulación". Nómadas. Número Especial de América Latina. España. Universidad Complutense de Madrid, 2013: 161-174.

TESIS

Aguilar Aguilar, Arturo. "El turismo de cruceros como elemento en la transformación del paisaje de Cozumel". [Tesis de Maestría en Gestión Sustentable del Turismo]. México: Universidad de Quintana Roo, 2014.

Martínez, Claudia. "Tipología de los sitios con uso turístico y recreativo en la Isla de Cozumel, México". [Tesis de Maestría en Geografía]. México: Universidad Nacional Autónoma de México, 2007. 
DOCUMENTOS ELECTRÓNICOS

Cruise Line International Association Inc (cLIA). The overview. exuu, Fort Lauderdale: Cruise Line International Association Inc. 2011. En: <http://cruising.org/ sites/default/files/misc/2011 Finalov.pdf> [consultado el 26 de mayo de 2014].

Enríquez Acosta, Jesús Ángel. "Las nuevas ciudades para el turismo. Caso puerto Peñasco, Sonora, México". X Coloquio Internacional de Geocrítica. Diez años de cambios en el mundo, en la Geografía y las Ciencias Sociales, 1999-2008. Universidad de Barcelona, 26-30 de mayo de 2008. En: <http://www.ub.edu/ geocrit/-xcol/63.htm>_[consultado el 26 de mayo de 2014].
Fondo Nacional de Fomento al Turismo. Nuevos Megaproyectos. México, 2008. En: <http://www.fonatur.gob.mx/ es/proyectos_desarrollos/cozumel/> [consultado el 26 de mayo de 2014].

OTROS

Instituto Nacional de Estadística, Geografía e Informática (INEGI).Censo de población y vivienda 2010. México, 2010.

Organización Mundial del Turismo (омт). Turismo de cruceros; situación actual, España, 2008.

Fecha de ingreso: 02/06/2014 Fecha de aprobación: 25/01/2015 
